\title{
THE FLUXGATE MAGNETOMETER SIMULATION IN COMSOL MULTIPHYSICS
}

\author{
Andrey Kolomeytseva, Pavel Baranov and Ivan Zatonov \\ National Research Tomsk Polytechnic University, 634050, Tomsk, Russia
}

\begin{abstract}
This article describes the fluxgate magnetometer simulation in Comsol Multiphysics software package. The simulation results coincide with the experiment described earlier. Decomposition of the output signal by the Fourier coefficients shows a frequency doubling.
\end{abstract}

\section{Introduction}

Weak magnetic field measurement (from $10^{-11}$ to $10^{-4} \mathrm{~T}$ ) is a crucial objective in terms of space and geophysical research, attitude control [1-6], magnetic tomography, defectoscopy, non-destructive testing [7-10] etc.

A fluxgate magnetometer is the most commonly used to measure weak magnetic field. However, despite the high level of achieved characteristics, measurement accuracy improving is possible.

Electrostatic, electromagnetic, thermal and gas-dynamic interactions influence on fluxgate magnetometer characteristics. It is extremely difficult to solve this type of problems analytically. Fluxgate magnetometer successful development requires physical and simulation modelling more reliable predicting of the device behaviour. The solution to this problem is a finite-element simulation. It significantly reduces efforts to an experimental study and gives an understanding of the subject.

Modern systems of the finite-element simulation are able to implement a wide variety of fluxgate magnetometer design and evaluate their parameters. The need for such simulation is undeniable in the context of the search for new solutions. Nevertheless, there is no described technique for fluxgate magnetometer simulation with finite-element methods.

In this paper, we describe the main stages fluxgate magnetometer simulation in Comsol Multiphysics software package. The simulation input data is get from [11].

\section{Model definition}

Today, differential fluxgate magnetometers are the most common. Such a fluxgate magnetometer consists of two identical permalloy cores of rectangular cross-section that are parallel to each other. Around each core is a coil. They form excitation circuit. A secondary coil is wound on both primary coils. In a couple with indicator device, it forms a measuring circuit. Primary coil current $i$ induces magnetic fields in cores that is opposite in direction

\footnotetext{
${ }^{a}$ Corresponding author:aak65@tpu.ru
} 
to each other. Provided that the cores are identical and are in the external field $H_{0}$, magnetic flux density can be written as:

$$
\left\{\begin{array}{l}
B^{\prime}=B\left(H_{0}-H_{1}\right) \\
B^{\prime \prime}=B\left(H_{0}+H_{1}\right)
\end{array}\right.
$$

According to the Faraday's law of induction, output voltage in the secondary coil is:

$$
\mathrm{e}=-\mathrm{sw}_{2} \frac{\mathrm{d}}{\mathrm{dt}}\left(\mathrm{B}^{\prime}+\mathrm{B}^{\prime \prime}\right)
$$

We approximate $B=f(H)$ by a polynomial with degree 3 :

$$
\mathrm{B}=\mathrm{aH}+\mathrm{bH}^{3}
$$

where $a$ and $b-$ is the approximate coefficients. They depend on the material and shape of the core. obtain:

Assuming that $H_{1}=H_{m} \cdot \sin (\omega t)$ and $H_{0}=$ const, on substitution (3) into (2), we

where $\omega$-is excitation current frequency.

$$
\mathrm{e}=6 \omega \mathrm{bsw}_{2} \mathrm{H}_{0} \mathrm{H}_{\mathrm{m}}^{2} \sin 2 \omega \mathrm{t}
$$

It is seen from (4) that the output voltage has a doubled frequency and is proportional to the magnitude of the measured external field. The principles of the differential fluxgate magnetometer theory are described in the presented equations.

The model created by us consists of two cores, two primary coils and a measuring coil (Figure 1). Each primary coil is wound on a core. The measuring coil encircles both primary coils.

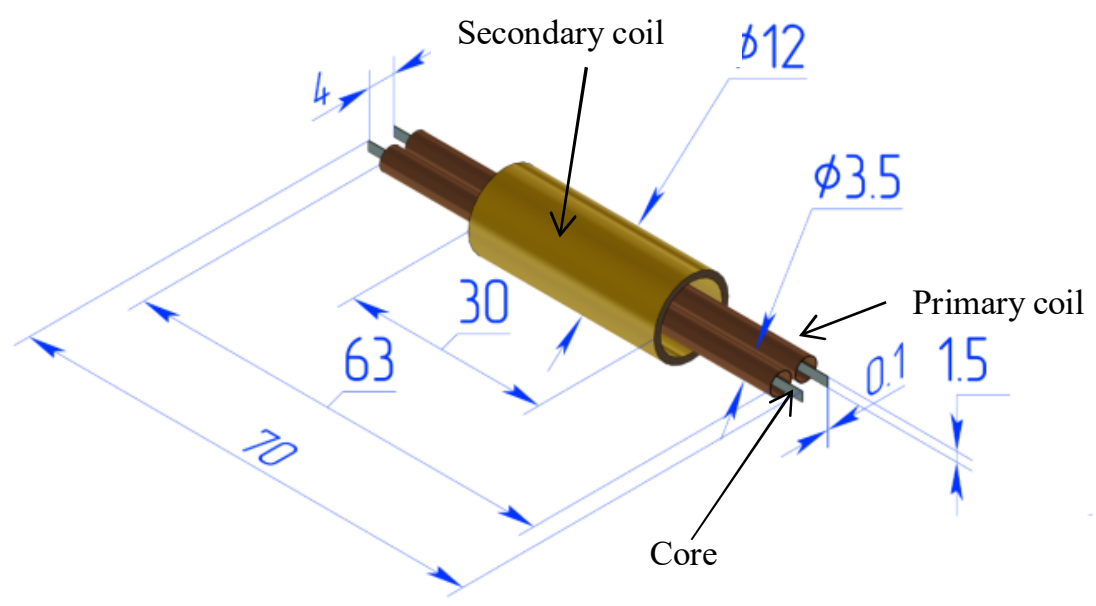

Fig. 1. Model illustration of a fluxgate magnetometer.

\subsection{Electric circuit}

The model assumes that the primary and secondary coils are made of thin wire and consist of many turns. The primary coils have their own active resistance $R_{p}$ and are connected to an alternating current source. The primary and secondary coils are inductively coupled, as shown in Figure 2. The secondary coil also has its own active resistance $R_{s}$. 


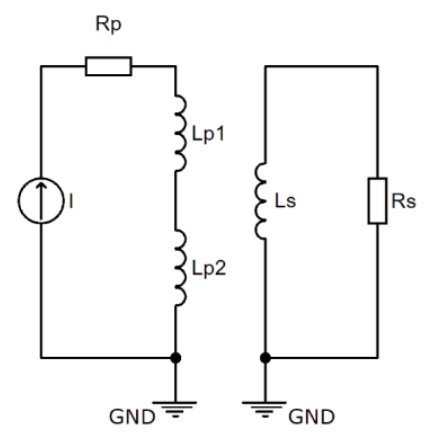

Fig. 2. Current source connected with a primary coil.

The current source generates sine signal with a frequency of $1.5 \mathrm{kHz}$ and amplitude of $20 \mathrm{~mA}$. The primary coil consists of 315 turns. Each primary coil has an active resistance of $4.6 \mathrm{ohms}$. The secondary coil consists of 2450 turns and its active resistance is $530 \mathrm{ohms}$.

The measured magnetic field is directed along the fluxgate magnetometer sensitivity axis and has a value of $10^{-4} \mathrm{~T}$.

\subsection{Materials}

The air cylinder is around the fluxgate magnetometer. Copper from Material Library is selected for the coils. Nickel Steel Square 50 is chosen as the core material. Jiles-Athertron model describes non-linear material characteristics. Due to this, it is possible to take into account the hysteresis in the core. The parameters of the Jiles-Atherton model are determined experimentally. The core material parameters are summarized in Table 1.

Table 1. Core material parameters

\begin{tabular}{|l|c|c|}
\hline Property & Value & Unit \\
\hline Electrical conductivity & 2.088 & $\mathrm{MS} / \mathrm{m}$ \\
\hline Relative permittivity & 1 & 1 \\
\hline Maximum magnetization parameter & 1.6 & $\mathrm{MA} / \mathrm{m}$ \\
\hline Langevin slope parameter & 560 & $\mathrm{~A} / \mathrm{m}$ \\
\hline Pinning parameter & 1200 & $\mathrm{~A} / \mathrm{m}$ \\
\hline Reversibility parameter & 0.1 & 1 \\
\hline Interdomain coupling parameter & 0.0007 & 1 \\
\hline
\end{tabular}

\subsection{Mesh}

Correct adjustment of a mesh is great importance in simulation. When studying fluxgate magnetometer, it is crucial that the cores must interact with the field equally. In this paper, we set up the mesh each domain manually. Thereby, the mesh becomes uniform and domains are more symmetrical than with the physics-controlled mesh. The resulting mesh is shown in Figure 3. 


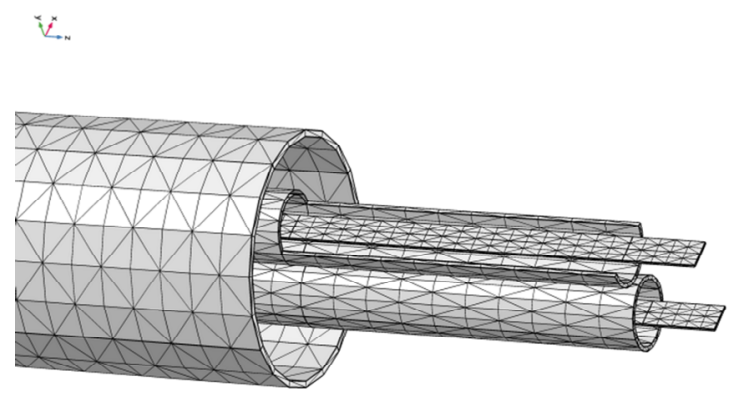

Fig. 3. Finalized mesh.

\section{Results and discussion}

The Figure 4 shows the distribution of magnetic flux density in the cores and the currents direction at $t=0, t=\frac{T}{4}, t=\frac{T}{2}$ and $t=\frac{3 T}{4}$.

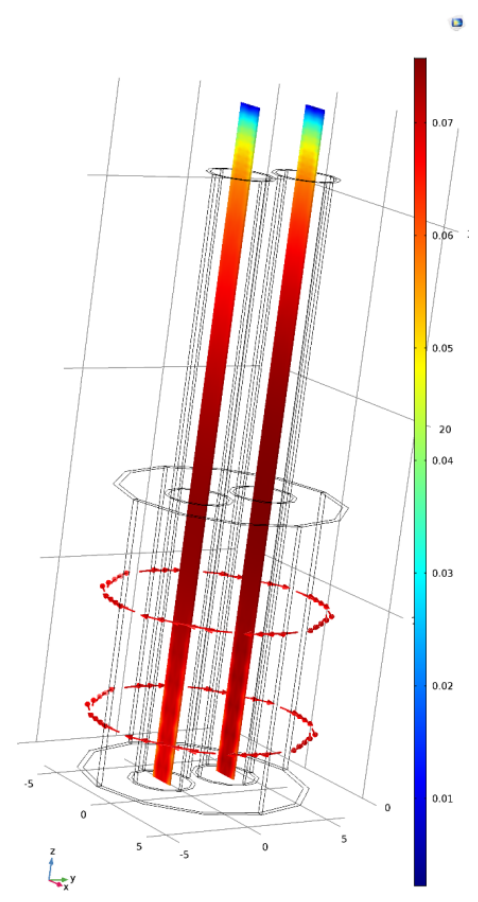

(a) $t=0$

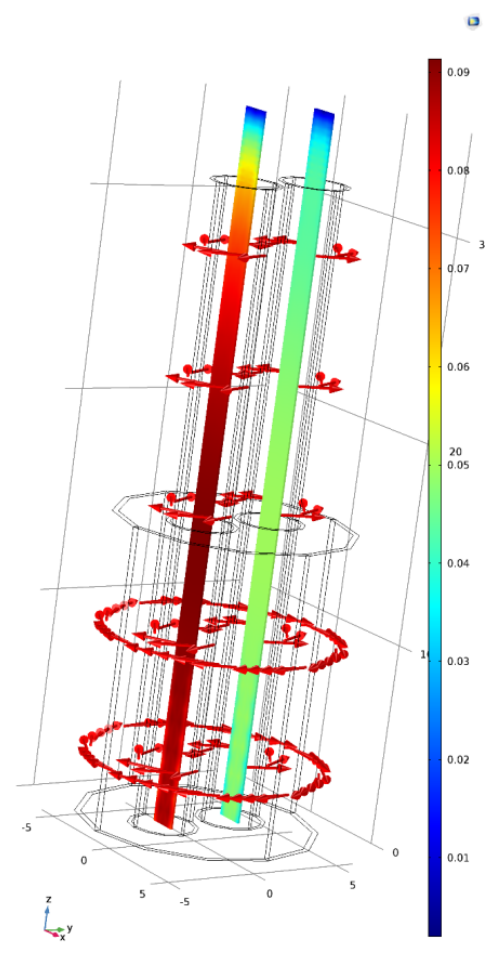

(b) $t=\frac{T}{4}$ 


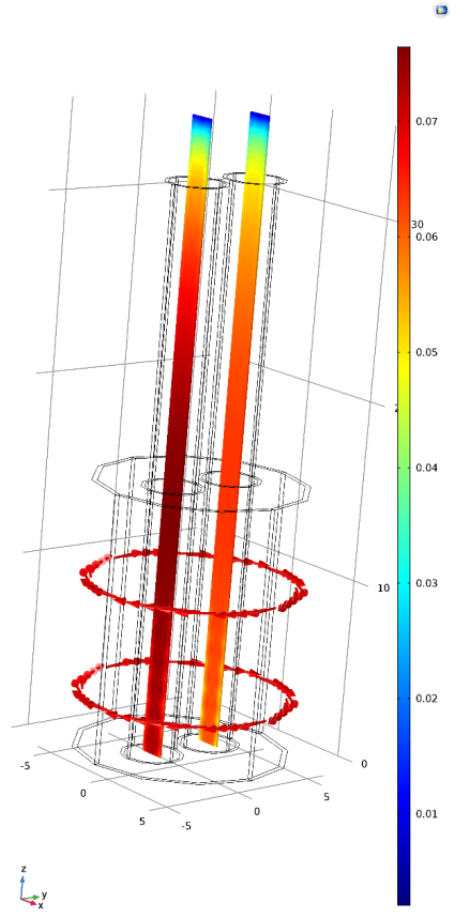

(c) $t=\frac{T}{2}$

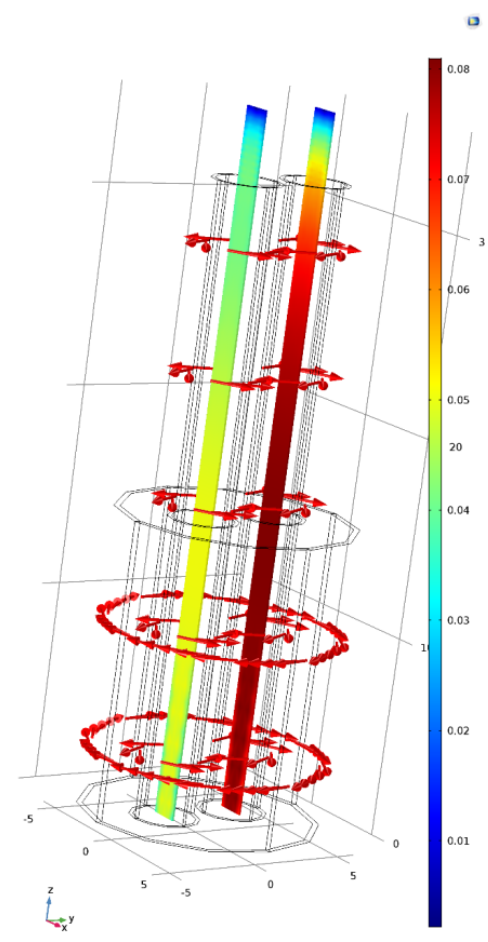

(d) $t=\frac{3 T}{4}$

Fig. 4. Magnetic flux density in cores (scaled) and current direction (red arrows).

The Figure 5 shows the current against time in the primary (a) and secondary coils (b).

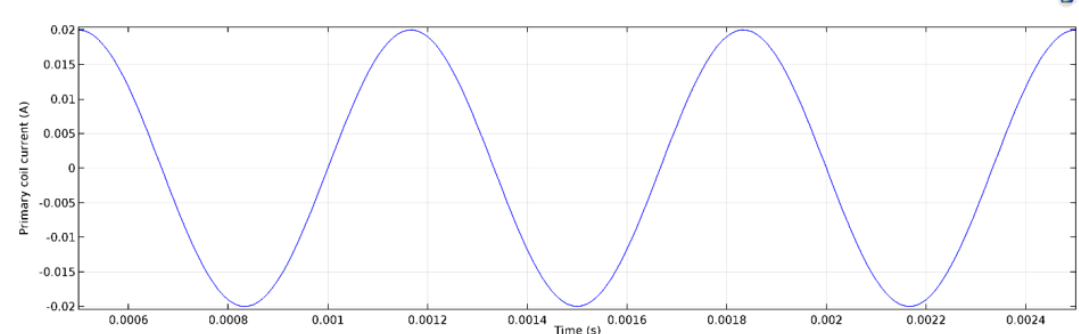

(a)

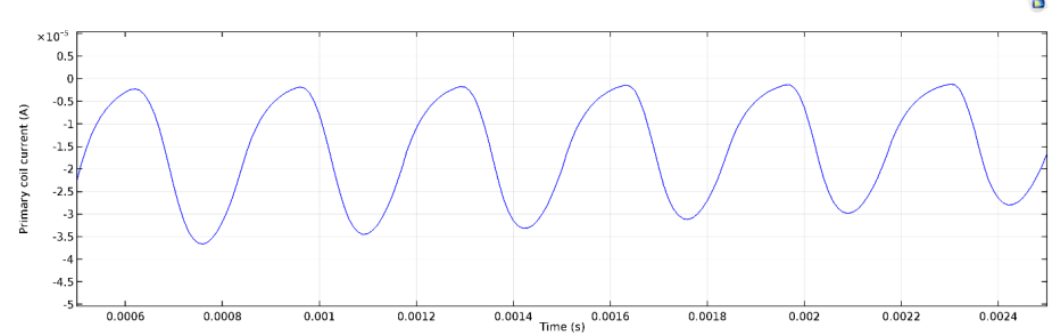

(b)

Fig. 5. Primary and secondary coil current timing diagram. 
The waveform coincides with the results of the experiment described in [11]. The output voltage has an error of $15-20 \%$. This can be explained by the difference between the core material used in the simulation and the experimental studies.

Decomposition into Fourier coefficients confirms the presence of a pronounced second harmonic in the output signal (Figure 6).

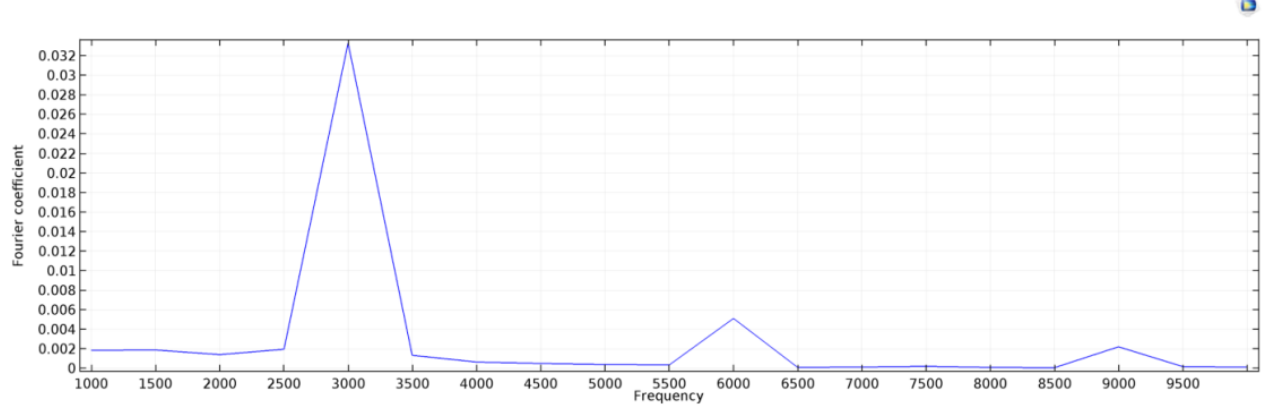

Figure 6. Factorization Fourier coefficients.

\section{Conclusion}

The paper describes the simulation main stages of a fluxgate magnetometer in Comsol Multiphysics software package. The result confirms the possibility of simulating similar measuring device by finite element methods. The shape and amplitude of the output signal in the measuring coil coincide with sufficient accuracy with the results of experimental studies performed by other authors. In the future, we plan to carry out a construction thermal analysis for the purpose of calculation the heat release, depending on the shape of the excitation signal.

\section{Acknowledgments}

The research is funded from Russian Science Foundation (RSF), Grant Number 17-7910083.

\section{References}

[1] P. Ripka, Magnetic Sensors and Magnetometers (Artech House, Boston, 2001)

[2] F. Kaluza, A. Gruger, H. Gruger, Sens. Actuators A, Phys. 106,48(2003)

[3] P. Ripka, Sens. Actuators A, Phys. 33 (3), 129 (1992)

[4] P. Ripka, IEEE Sens. J. 10(6), 1108 (2010)

[5] B. Andta, S. Baglioa, A. R. Bulsarab, C. Trigonaa Sens.\&Actuat. 151,145 (2009)

[6] S. Uchaikin, A. Likhachev, F. Cioata, et al., LT26 (2011)

[7] M. Janosek, P. Ripka, Sens.\&Actuat. 151,141(2009)

[8] W. Magnes, M. Díaz-Michelena, IEEE Trans. on Magnet. 45(10), 17 (2009)

[9] M. Vopàlensky, P. Ripka, A. Platil, Sens. Actuators. 106, 38(2003)

[10] V. Baranova, et al., XXI IMEKO, 4 (2015)

[11] U. Afanasiev, Ferrozondovie pribory (Energoatomizdat, Leningrad,1986) 